\title{
Removal of Blocking Artifacts using Various Filtering Techniques
}

\author{
Parmjeet Kaur \\ Assistant Professor, Department of Electronics and \\ Communication Engineering, \\ College of Engineering and Management,
}

Kapurthala.

\begin{abstract}
Image processing is the method for processing various kinds of images, processed images can be stored conveniently and transmission of such kind of images from one place to another place becomes easy to the user. By using image compression method we are able to represent the image with lesser number of bits. By using image compression technique we can reduce the bandwidth and the volume of the data to be transmitted. Different types of images can be processed and we can reduce blocking artifacts up to acceptable level. Some standard techniques MPEG and JPEG are used in image processing field. Some other types of images like GIF, JPG and PNG are used. Lossless compression is used for medical image compression because whole information is necessary for analysis; even a single bit loss is not bearable. Lossy image compression technique is used in photographic images because loss of bits is bearable. When we compress any kind of image by lossy type of image compression technique then there will be loss of bits, and when we want to recover such kind of image then we face problem of blurring of images, which is sometimes called as the annoying problem of blocking artifacts. To recover original image back we can use decompression followed by the different filtering methods. We can compare these filtering techniques on the basis of the MATLAB simulation results. Various Parameters can be compared like PSNR, MSE and BER.
\end{abstract}

\section{General Terms}

Image compression, Redundancy, compression factor, blocking artifacts, filtering techniques, removing noise et. al.

\section{Keywords}

DCT, Spatial filtering, hybrid filtering, JPEG, blocking artifacts, MSE, BER, PSNR.

\section{INTRODUCTION}

Image compression is the method of reducing the size and number of bytes required to represent a graphics file without sacrificing the quality of the image. By reducing the size of the file it becomes easy to save this type of files on small space of disk, and large number of images can be stored on small memory space. It also reduces the time required for images to be sent over the Internet or downloaded from Web pages.[1] The need for image compression becomes apparent when number of bits per image are computed resulting from typical sampling rates and quantization methods. Number of bits required to represent the information in an image can be reduced by removing the redundancy present in it. There are three types of redundancies. Two types of compression techniques are used, Lossless and Lossy compression. In lossless compression scheme, the reconstructed image, after compression, is identical to the original image. However it can achieve

\author{
Poonam Sethi \\ Assistant Professor, Department of Electronics and \\ Communication Engineering, \\ DAV Institute of Engineering and Technology, \\ Jalandhar
}

only modest amount of compression. Lossless compression is used for archival purposes, medical imaging, technical drawings, clip art or comics [2]. This is due to lossy compression methods, especially when used at low bit rates, introduce compression artifacts. An image reconstructed following lossy compression contains degradation relative to the original. This happens due to the compression scheme completely ignore redundant data. But lossy schemes are capable of acquiring much higher compression. Lossy methods are used in that application where small number of bits loss is acceptable. There are some lossy compressions techniques which produce minor differences can be called as virtually lossless. Image files can be compressed by many different ways. [3]Text file, image and program can be compressed but only up to some extent. This is known as lossless compression. There is no critical point up to which compression works perfectly. When there is some tolerance for loss, the compression factor can be greater than it can when there is no loss tolerance. For this reason, graphic images can be compressed more than text files or programs. Block diagram for processing of image is given below:

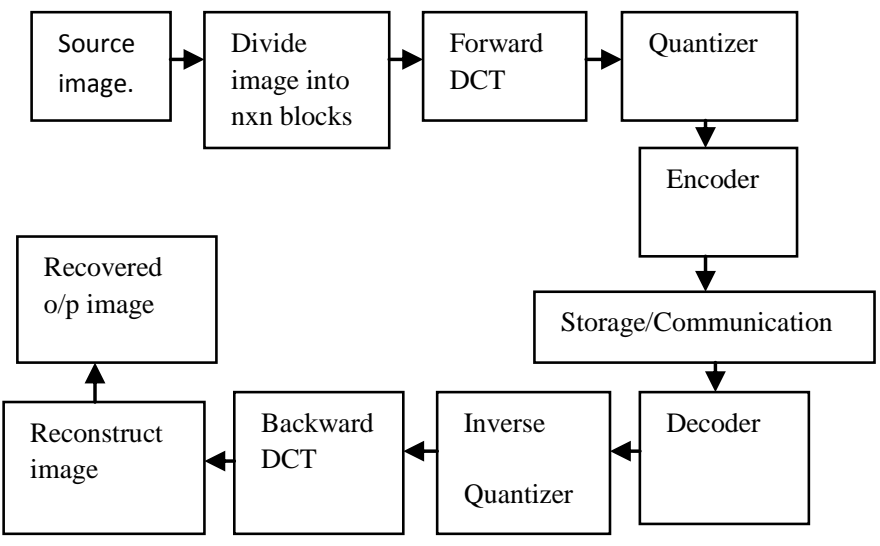

Figure1: Block diagram showing Compression and Decompression

2.1 Transformer: It converts input data from one format to another to reduce inter pixel redundancies in the input image. The success of the transform-based coding schemes is depends upon this thing that many of the resulting coefficients for most natural images have small magnitudes and can be quantized without causing significant distortion in the decoded image.[5] If the capability of compression information in fewer coefficients is higher, than the performance is better. 
2.2 Quantizer: With some existing fidelity criterion the accuracy of the transformer's output decreases by it and also reduces the psycho visual redundancies of the input image. We can't reverse this operation and must be omitted if lossless compression is desired. The quantization stage is at the core of any lossy image encoding algorithm. Quantization at the encoder side means partitioning of the input data range into a smaller set of values at the encoder side.

2.3 Entropy encoder: It creates a fixed or variablelength code to represent the quantizer output and maps the output in accordance with the code. Mostly, a variablelength code is used. This encoder further compresses the compressed values acquired by the quantizer to give more accurate output of the compression.

\section{IMAGE COMPRESSION USING DCT}

DCT separates images according to their different frequencies where less important frequencies are discarded by quantization and more important frequencies are used to retrieve the image during decompression. By transformation, DC T has many advantages. [1] It has been implemented in single integrated circuit. DCT can pack most information in fewest coefficients. It reduces the block like appearance called blocking artifact that results when boundaries between sub-images become visible.

When the input to the DCT equation is an M X N matrix, the equation treats each input column as an independent channel containing $\mathrm{M}$ consecutive samples. The equation outputs an $\mathrm{M} X \mathrm{X}$ matrix whose 1 the column contains the length$\mathrm{M}$ DCT of the corresponding input column.

$$
y(k, l)=w(k) \sum_{m=1}^{M} u(m, l) \cos \frac{\pi(2 m-1)(k-1)}{2 M} \ldots . .(1)
$$

$$
w(k)=\left\{\begin{array}{lc}
\frac{1}{\sqrt{M}}, & k=1 \\
\sqrt{\frac{2}{M}}, & 2 \leq k \leq M
\end{array}\right.
$$

The IDCT equation computes the inverse discrete cosine transform across the vector dimension of the input. For all other N-D input arrays, the equation computes the IDCT across the first dimension. The size of the first dimension frame size in this system must be a power of two.

When the input is an $M \mathrm{X} N$ matrix, then each input column is treated as an independent channel containing $M$ consecutive samples.

The equation outputs an $M \times N$ matrix whose $l$ the column contains the length- $M$ IDCT of the corresponding input column.

$y(m, l)=\sum_{k=1}^{M} w(k) u(k, l) \cos \frac{\pi(2 m-1)(k-1)}{2 M} \ldots .$.

The two-dimensional DCT for an input image A and output image B is given below. [4] The two-dimensional transform is equivalent to a one-dimensional DCT performed along a single dimension and by a one-dimensional DCT in the other dimension.

$$
\begin{aligned}
& B_{p q}=\alpha_{p} \alpha_{q} \sum_{m=0}^{M-1} \sum_{n=0}^{N-1} A_{m n} \cos \frac{\pi(2 m+1) p}{2 M} \cos \frac{\pi(2 n+1) q}{2 N}, \begin{array}{l}
0 \leq p \leq M-1 \\
0 \leq q \leq N-1
\end{array} \ldots(3) \\
& \alpha_{p}=\left\{\begin{array}{lc}
\frac{1}{\sqrt{M}}, \quad p=0 \\
\sqrt{\frac{2}{M}}, & 1 \leq p \leq M-1
\end{array} \alpha_{q}= \begin{cases}\frac{1}{\sqrt{N}}, & q=0 \\
\sqrt{\frac{2}{N}}, & 1 \leq q \leq N-1\end{cases} \right.
\end{aligned}
$$

$\mathrm{M}$ and $N$ are the row and column size of $\mathrm{A}$. The result is real if DCT is applied to real data. The DCT is used to concentrate information for making it useful for image compression applications.Idct 2 computes the two-dimensional inverse DCT using:

$$
A_{m n}=\sum_{p=0}^{M-1 N-1} \sum_{q=0} \alpha_{p} \alpha_{q} B_{p q} \cos \frac{\pi(2 m+1) p}{2 M} \cos \frac{\pi(2 n+1) q}{2 N}, \begin{gathered}
0 \leq m \leq M-1 \\
0 \leq n \leq N-1
\end{gathered}, \ldots(4)
$$

\section{LITERATURE STUDY}

There have large numbers of researches on image compression and blocking artifacts reductions. There are number of filtering techniques which can be used in image processing field. An advanced discrete cosine transform based image compression method that combined advantages of several approaches is used. Image compression using Discrete Cosine Transform is one of the simplest commonly used compression methods. The literature study is given below:

Nikolay N. Ponomarenko et al. in 2007, [1] presented an advanced discrete cosine transform (DCT)-based image compression method that combined advantages of several approaches. Sukhwinder Singh et al. in same year invented a 2D step function method, between two neighbouring blocks. The blocking artifacts were detected by using block activity based on human visual system (HVS) and block statistics. [2]The boundary regions between blocks were identified as either smooth or non-smooth regions. The blocking artifacts in smooth regions were removed by modifying a few DCT coefficients appropriately. F. M. Bayer et al. in 2010 introduced a numerical approximation method for the DCT based on round-off techniques. [3] This method was a multiplier less technique with low arithmetic complexity. They gave emphasis to approximating the 8-point DCT. They presented that in several scenarios. Ee-Leng Tan1 et al. in 2008 presented a computational scalable approach to resize images by factors of $\mathrm{P} / \mathrm{Q} \times \mathrm{R} / \mathrm{S}$ in the discrete cosine transform (DCT) domain. [5] Their resizing approach was based on the frame work which employed spatial relationship between a DCT block and its sub-blocks. Sang-Jun Parket et al. in 2010 presented a direct image resizing method in the compressed domain. [6] This method omitted the inverse discrete cosine transform (IDCT) operation required to represent the image in the spatial domain.Wang Ruiet al. in 2007 proposed a hybrid method based on anisotropic diffusion (AD) for ultrasound speckle suppression and edge enhancement. [7]This method was designed to utilize the different denoising properties of three techniques: median filtering, improved AD filtering and isotropic diffusion filtering.I. O. Kirenko et al. in 2006 presented a method for artifacts reduction of MPEG compressed video sequences. [8]This method was used to remove such blocking artifacts blockiness, ringing, mosquito 
noise and quantized film and grain. Parveen Kumar et al. in 2011 presented that the reconstructed images from JPEG compression produced noticeable image degradation near the block boundaries, in particular for highly compressed images, because each block was transformed and quantized independently. Ju Jia Zou et al. [10] presented that a deblocking method based on projection onto convex sets (POCS) is proposed to reduce blocking artifacts in compressed images coded by the block discrete cosine transform. M. I. Khalil et al in 2010 presented that digital images contain large amount of information that need evolving effective techniques for storing and transmitting the ever increasing volumes of data. [11] Image compression addresses the problem by reducing the amount of data required to represent a digital image. Janaki. R et al. in 2012 presented that Medical image compression applications are quality-driven applications which demand high quality for certain regions that have diagnostic importance in an image, [13] where even small quality reduction introduced by lossy coding might alter subsequent diagnosis, which might cause severe legal consequences. Due to this, lossless techniques have been extensively used by them. Fang Zhu et al. 2011 presented that Blocking artifacts are significant problem in DCT-based image compression, [14] which introduce the apparent distortion of visual quality. In this paper, they proposed blocking artifacts deduction method. Robert Ferstl et al. in 2007 invented an article on the ideas behind a few programs we developed for spatial data analysis in EViews and MATLAB. Dr. H. B. Kekre et al. in 2011 presented Iris recognition systems, these were unavoidable in emerging security and authentication mechanisms. They made a comparative study of performance of image transforms Discrete Cosine Transform (DCT), Haar wavelet and DCT wavelet. V. Santhi et al. in 2009 proposed watermarking techniques and information hiding techniques.[18]Digital watermarking is a technique in which a piece of digital information is embedded into an image and extracted later for ownership verification. Zhi-Heng Zhou et al. in 2006 proposed that the block-based DCT compression methods usually result in discontinuities called blocking artifacts at the boundaries of blocks due to the coarse quantization of the coefficients. [19] They invented a new blocking artifacts reduction algorithm is proposed to reduce blocking artifacts and preserve edges adequately.

\section{Comparison parameters of images 5.1Mean Square Error (MSE):}

When we consider two images I and $\mathrm{K}$ mxn monochrome type. So that one of two images is considered a noisy approximation of the other. Formula for MSE is given below:

$$
M S E=\frac{1}{m n} \sum_{i=0}^{m-1} \sum_{j=0}^{n-1}[I(i, j)-K(i, j)]^{2}
$$

\subsection{Peak Signal-To-Noise Ratio (PSNR):}

It is a term for the ratio between the maximum possible power of a signal and the power of unwanted noise that affects the fidelity of its representation. Because many signals have a very wide dynamic range, PSNR is usually expressed in terms of the logarithmic decibel scale. Its unit is decibel $(\mathrm{dB})$.

$$
P S N R=10 \cdot \log _{10}\left(\frac{M A X_{I}^{2}}{M S E}\right)
$$

Here, $M A X_{I}$ is the maximum possible pixel value of the image, and MSE is the Mean Square Error. If we have $B$ bits per sample, $M A X_{I}$ is $2^{B}-1$. When the two images are identical, the MSE will be zero. For this value the PSNR is undefined.

5.3 Bit-Error-Rate (BER): We determine how many of the received bits are in error.

$\mathrm{BER}=$ te/length $(\mathrm{tx})$

where 'te' is the total number of bit errors, and ' $t x$ ' is the transmitted bit vector.

\section{VARIOUS FILTERING TECHNIQUES}

\subsection{Dct Filtering}

DCT is a Fourier-related transform similar to the discrete Fourier transform (DFT), but using only real numbers. DCTs are equivalent to DFTs of roughly twice the length, operating on real data with even symmetry, while in some variants the input and output data are shifted by half a sample. The DCT is used in JPEG image compression and MPEG. There, the twodimensional DCT-II of $\mathbf{N} \times \mathbf{N}$ blocks is computed and the results are quantized and entropy coded.

\subsection{Spatial Filtering}

Spatial filtering is a term used to describe the methods used to compute spatial density estimates for events observed at individual locations. Spatial prediction does not out-perform pure DCT based technique in terms of bit-rate tradeoff. At very low bit rates it results in far fewer blocky artifacts and better visual quality. It describes a set of tools for displaying functions estimated from these data points that are distributed in two-dimensional space. This method used a twodimensional filter in the areas away from edges, and for near edges, one-dimensional filter aligned parallel to edge so as to minimize the blocking artifacts.

\subsection{Hybrid Filtering}

Hybrid filtering technique tries to combine both the methods discussed above. It does not require IDCT; its implementation in hardware becomes easy. Due to the significant reduction of the ringing effect, this method yields better performance in terms of both objective and subjective views than the other methods. This method of hybrid approach is applied to the blocks with vertical or horizontal edge. It is said to improve processing speed, suppress speckle and enhance edges. 

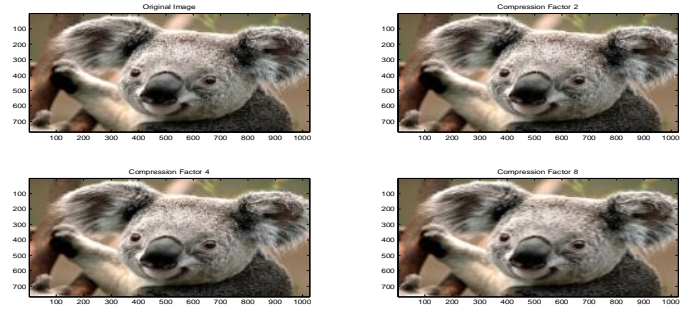

Figure 2: Up-left: Original image Koala.jpg, Up-right: Image with compression ratio 2, Bottom-left: Image with compression ratio4, Bottom-right: Image with compression ratio8.
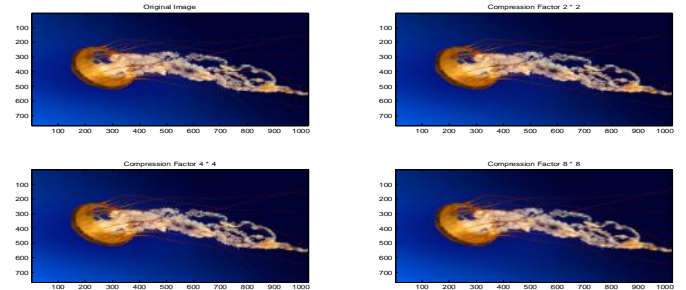

Figure 4: Up-left: Original image Jellyfish.jpg, Up-right: Image with compression ratio 2, Bottom-left: Image with compression ratio 4, Bottom-right: Image with compression ratio8.
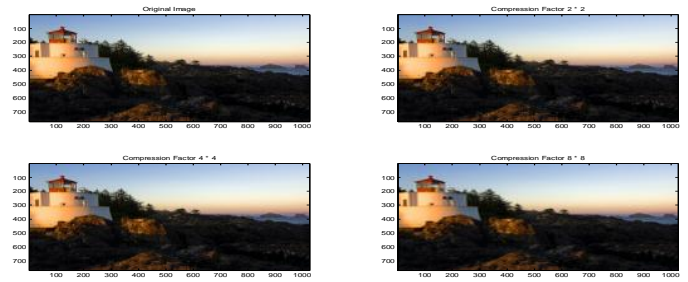

Figure 6: Up-left: Original image Lighthouse. Jpg, Up-right: Image with compression ratio 2, Bottom-left: Image with compression ratio 4, Bottom-right: Image with compression ratio8.
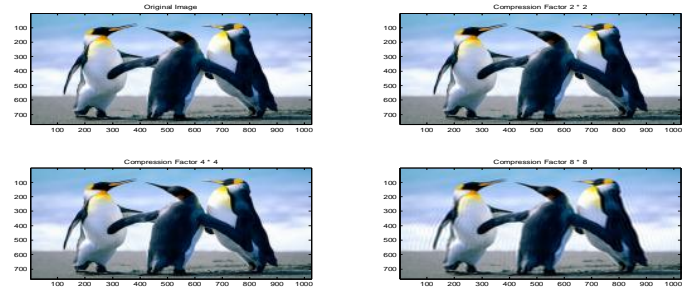

Figure8: Up-left: Original image Penguins. Jpg, Up-right: Image with compression ratio 2, Bottom-left: Image with compression ratio 4, Bottom-right: Image with compression ratio8.
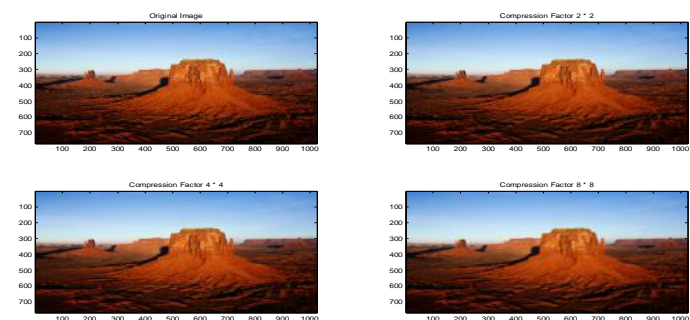

Figure3: Up-left: Original image Desert. Jpg, Up-right: Image with compression ratio 2, Bottom-left: Image with compression ratio 4, Bottom-right: Image with compression ratio8.
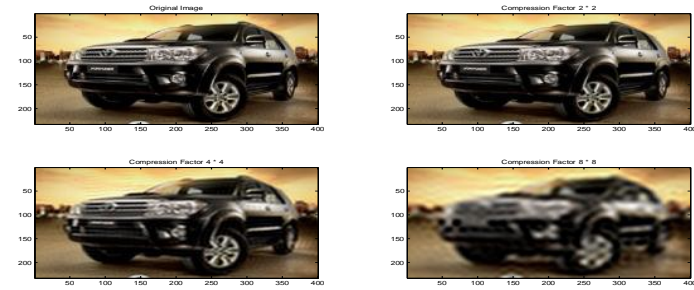

Figure5: Up-left: Original image Toyota_ Fotuner.jpg, Upright: Image with compression ratio 2 , Bottom-left: Image with compression ratio 4, Bottom-right: Image with compression ratio8.
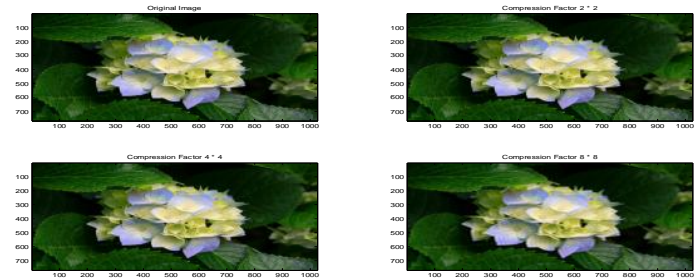

Figure 7: Up-left: Original image Hydrangeas.jpg, Up-right: Image with compression ratio 2, Bottom-left: Image with compression ratio 4, Bottom-right: Image with compression ratio8.
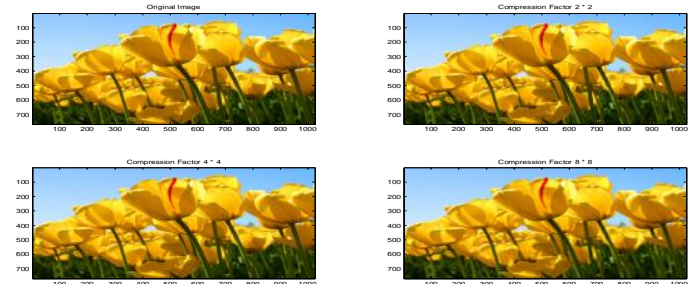

Figure 9: Up-left: Original image Tulips. Jpg, Up-right: Image with compression ratio 2, Bottom-left: Image with compression ratio 4, Bottom-right: Image with compression ratio8. 
Table1: Various images recovered by three different filtering Techniques.

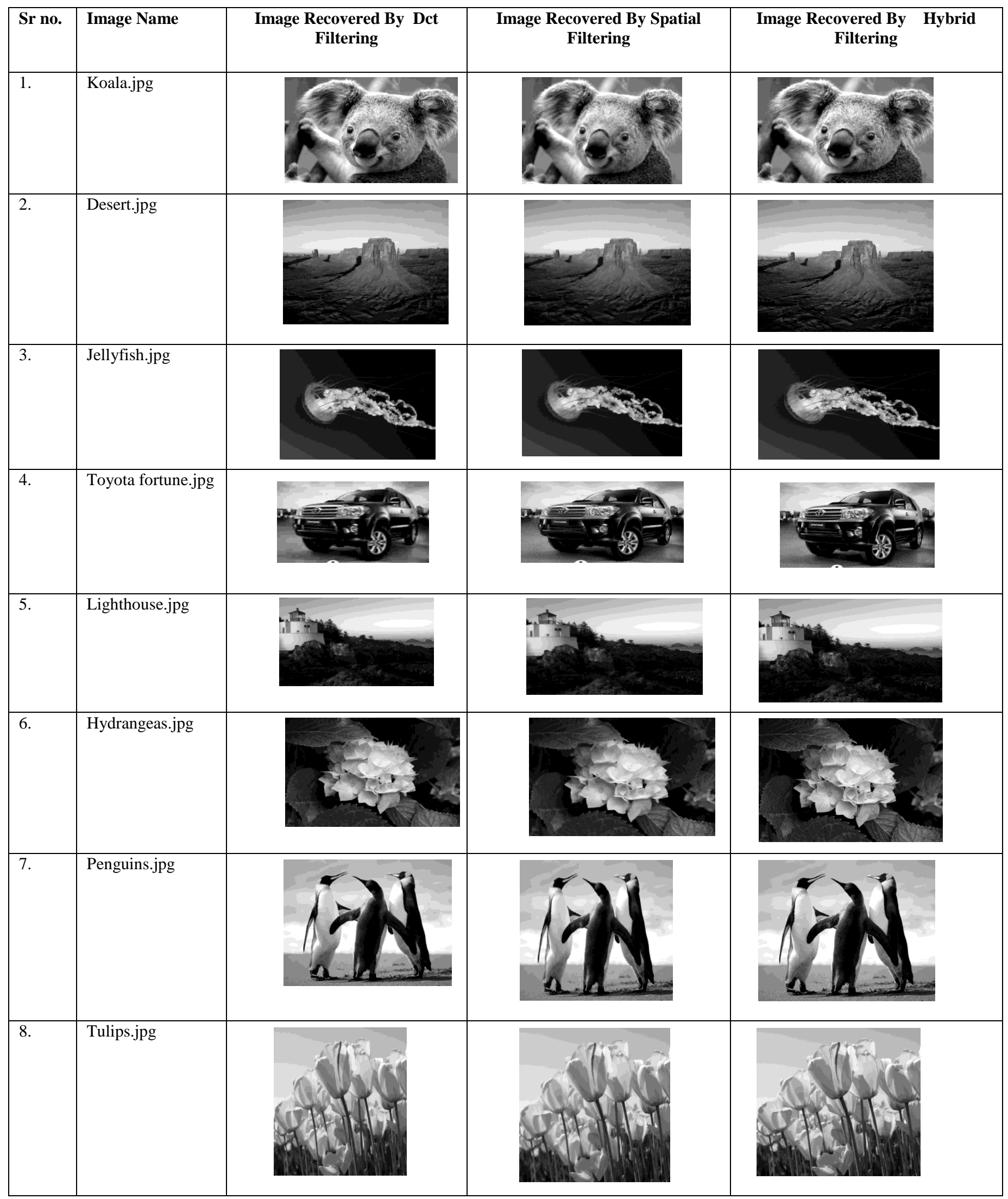


Table 2: Performance of the three Filtering techniques on various compressed images, Comparison of MSE, BER and PSNR

\begin{tabular}{|c|c|c|c|c|c|c|c|c|c|c|}
\hline \multirow[b]{2}{*}{ Sr no. } & \multirow[b]{2}{*}{ Image name } & \multicolumn{3}{|c|}{ DCT filtering } & \multicolumn{3}{|c|}{ Spatial filtering } & \multicolumn{3}{|c|}{ Hybrid Filtering } \\
\hline & & $\begin{array}{c}\text { M1 } \\
\text { MSE }\end{array}$ & $\begin{array}{c}\text { B1 } \\
\text { BER }\end{array}$ & $\begin{array}{c}\text { P1 } \\
\text { PSNR } \\
\end{array}$ & $\begin{array}{l}\text { M2 } \\
\text { MSE }\end{array}$ & $\begin{array}{l}\text { B2 } \\
\text { BER }\end{array}$ & $\begin{array}{l}\text { P2 } \\
\text { PSNR }\end{array}$ & $\begin{array}{l}\text { M3 } \\
\text { MSE }\end{array}$ & $\begin{array}{l}\text { B3 } \\
\text { BER }\end{array}$ & $\begin{array}{l}\text { P3 } \\
\text { PSNR } \\
\end{array}$ \\
\hline 1. & Koala.jpg & 33.3844 & 0.0304 & 32.8954 & 12.4253 & 0.0269 & 37.1877 & 0.5084 & 0.0196 & 51.0690 \\
\hline 2. & Desert.jpg & 25.8708 & 0.0294 & 34.0027 & 7.6210 & 0.0254 & 39.3107 & 0.5596 & 0.0197 & 50.6520 \\
\hline 3. & Jellyfish.jpg & 9.0835 & 0.0259 & 38.5433 & 3.9716 & 0.0237 & 42.1412 & 0.0529 & 0.0164 & 60.9000 \\
\hline 4. & Toyota_fortuner.jpg & 29.1840 & 0.0299 & 33.4749 & 11.5643 & 0.0267 & 37.4996 & 0.8284 & 0.0204 & 48.9485 \\
\hline 5. & Lighthouse.jpg & 17.3681 & 0.0280 & 35.7333 & 6.2726 & 0.0249 & 40.1563 & 0.3473 & 0.0190 & 52.7242 \\
\hline 6. & Hydrangeas.jpg & 15.5371 & 0.0276 & 36.2171 & 6.0090 & 0.0248 & 40.3428 & 0.2012 & 0.0182 & 55.0949 \\
\hline 7. & Penguins.jpg & 19.6921 & 0.0284 & 35.1879 & 5.7761 & 0.0247 & 40.5145 & 0.2377 & 0.0184 & 54.3702 \\
\hline 8. & Tulips.jpg & 12.0474 & 0.0268 & 37.3219 & 6.5132 & 0.0250 & 39.9928 & 0.1501 & 0.0177 & 56.3677 \\
\hline
\end{tabular}

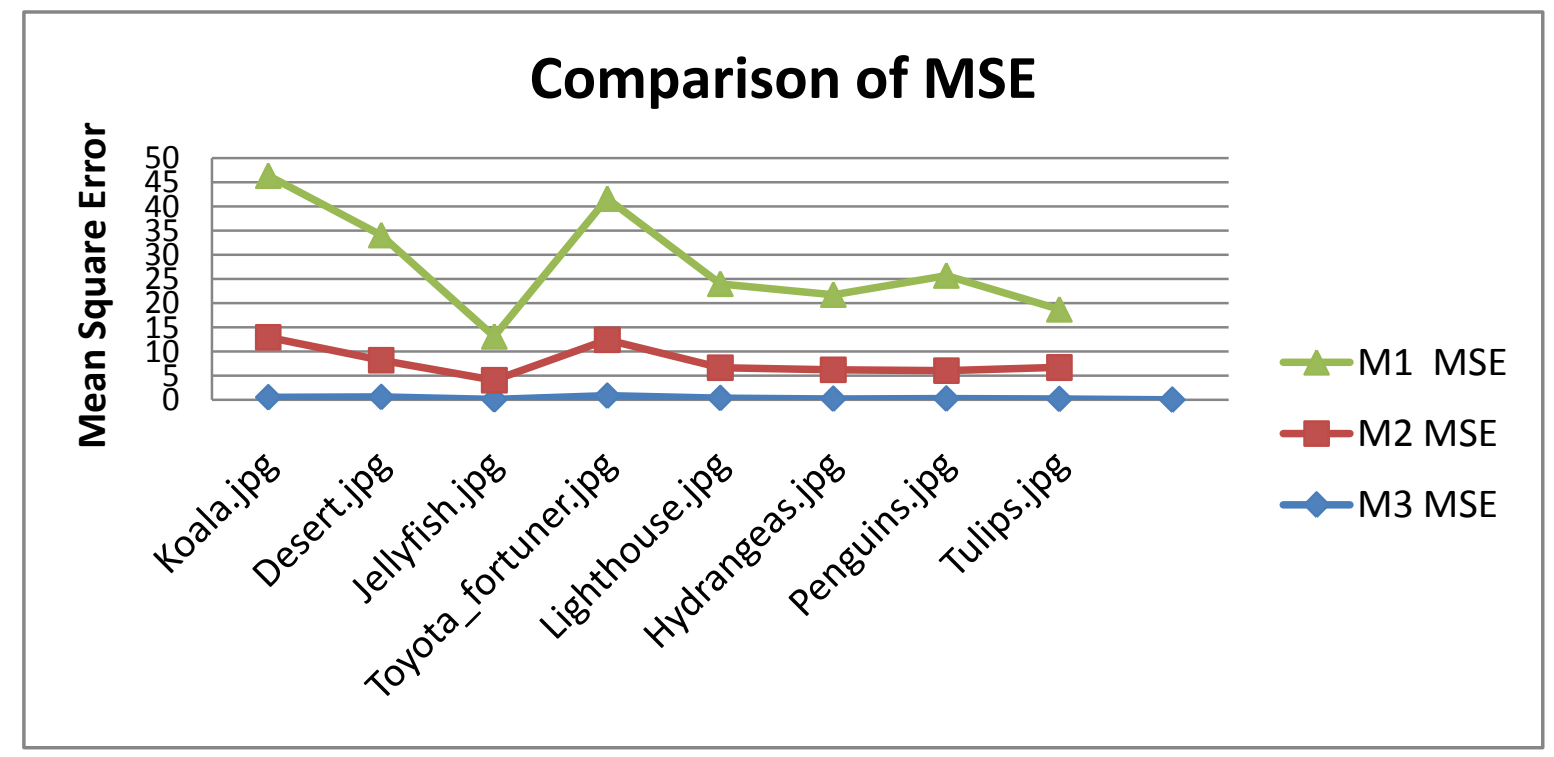

Figure 10: Graph Showing Comparison of Mean Square Error of The Different Images With Three Filtering Techniques.M1 is MSE For DCT Filtering, M2 is MSE For Spatial Filtering, M3 is MSE For Hybrid Filtering

\section{EXPERIMENTAL RESULTS}

After doing simulation on MATLAB R2010a version 7.10.0 software we observed that by taking different types of images having .jpg extension we can compare qualitatively and quantitatively various filtering techniques and compression factor change effect. Firstly we fetched different images of .jpg form. Then we take the original image one by one, and then compress these images by using DCT compression.
Results obtained after performing DCT compression of various orders on original images are shown. Images obtained after applying $8 \times 8$ DCT are shown in figure2 up to figure9. Various types of images are used by us and the results obtained depend upon the composition of the image, edges present in image color composition, size and type of image. The image after compression factor 2 will occupy less space as compared to original image. And as we increase the compression factor blurring of image increases but benefit is that space required to store it decreased. 


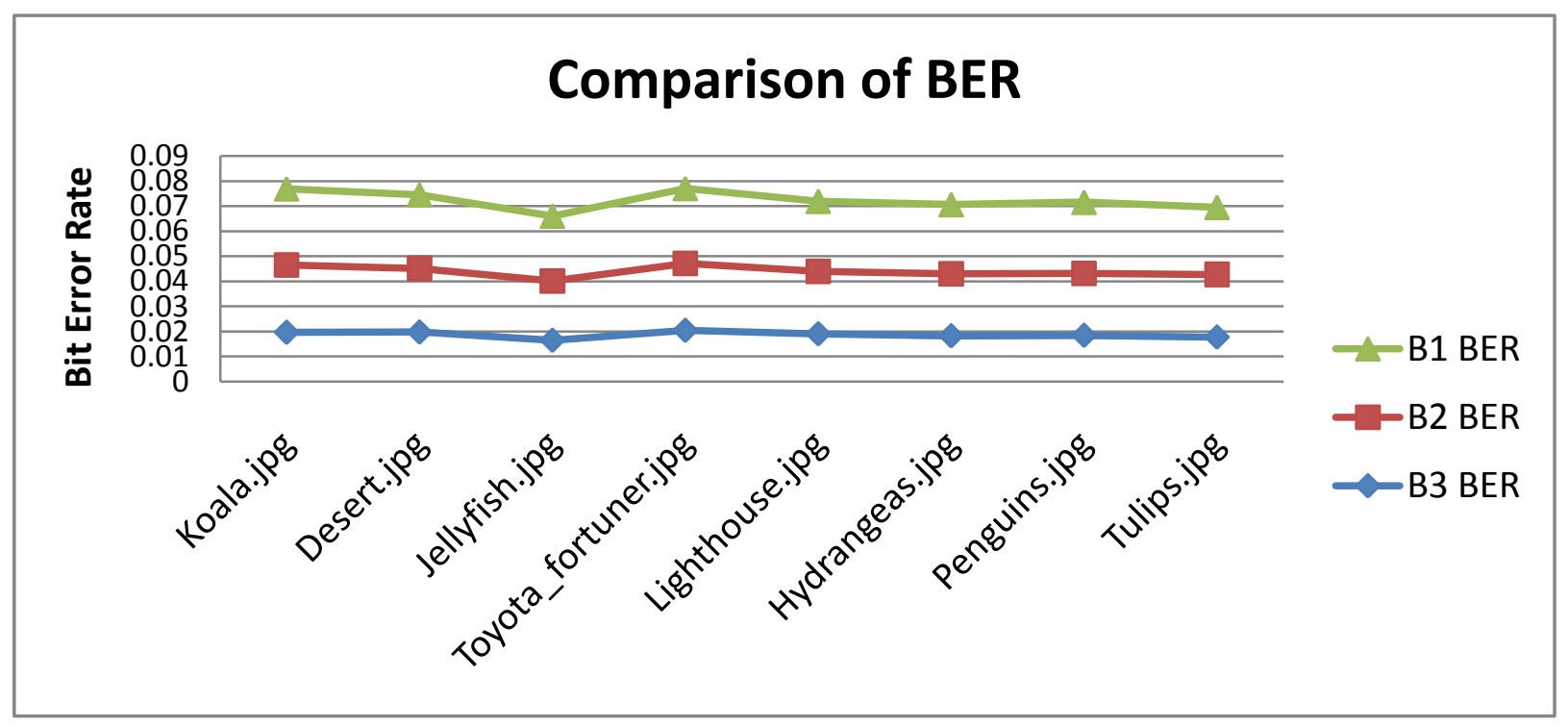

Figure11: Graph showing Comparison of Bit Error Rate of the Different Images With three Filtering techniques. B1 is BER for DCT Filtering, B2 is BER for Spatial Filtering, B3 is BER for Hybrid Filtering

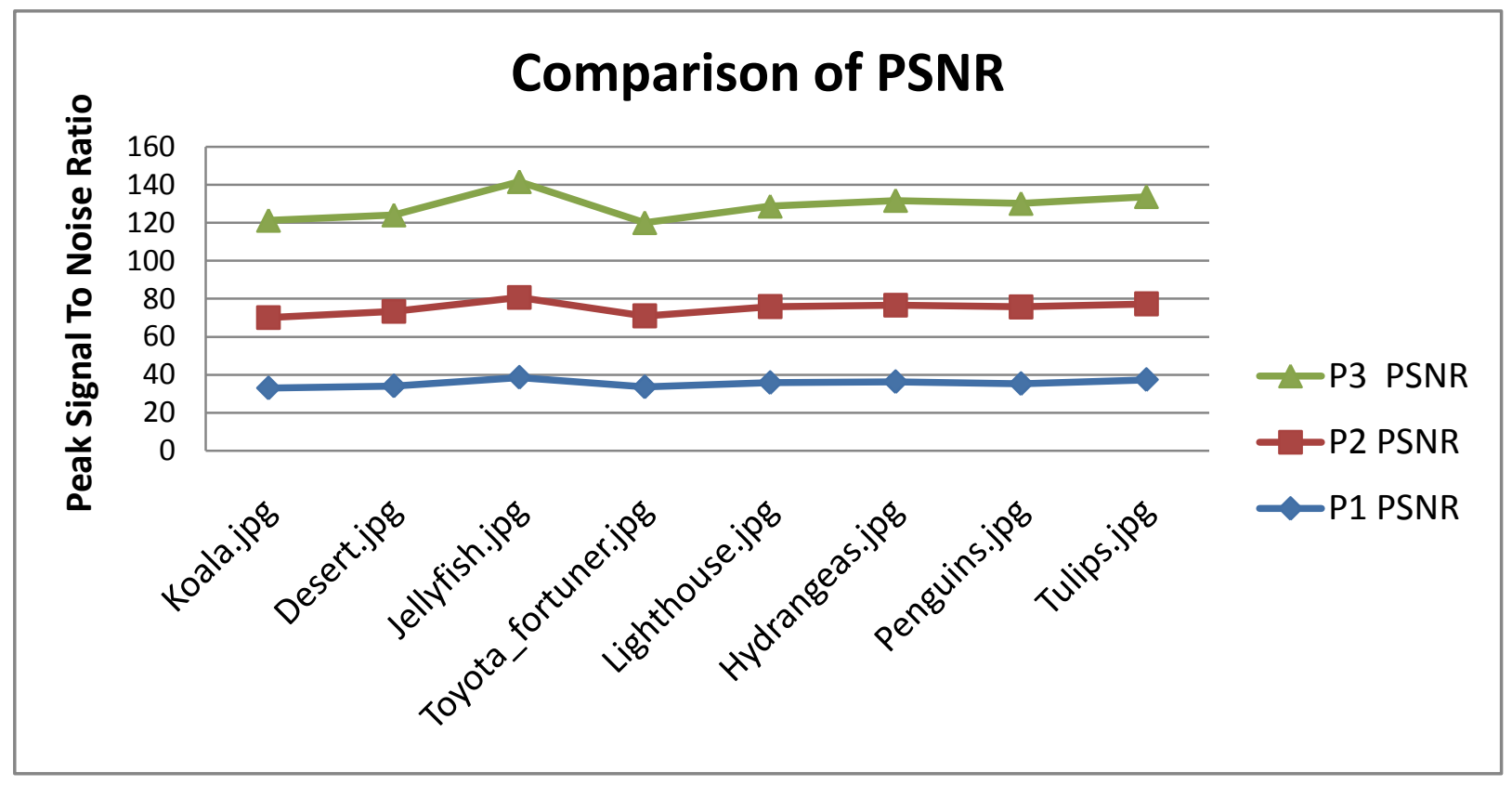

Figure12: Graph showing Comparison of Peak Signal to Noise Ratio of the different Images with three filtering techniques.P1 is PSNR for DCT Filtering, P2 is PSNR for Spatial filtering, and P3 is PSNR for Hybrid Filtering

So we did compression up to moderate rate that is compression factor 4 and 8 and results are shown diagrammatically From figure 2 to figure 9. This image when decompressed shows blocking artifacts in it. To remove those blocking artifacts we need to use the filtering techniques. So we used the DCT, Spatial and hybrid filtering technique. Experimental results compared the various parameters like BER, MSE, and PSNR in Decibel, of these three filtering techniques. Results are showing original and compressed images with different compression factor.
We concluded that as we increase the compression factor the blurring of the image increases. Table1.is showing various images recovered by three different filtering Techniques. Table2.is showing various parameters obtained using three different filtering techniques by MATLAB simulation, figure 10.is showing comparison of MSE of three filtering techniques, figure11. Is showing comparison of BER of three filtering techniques and figure12.is showing comparison of PSNR of three filtering technique. 


\section{CONCLUSION}

We concluded that both qualitatively and quantitatively the spatial filtering technique is better than the DCT filtering and hybrid filtering is better than the both filtering techniques. Because when we compare first two techniques then we observed that PSNR of second method is more than first and MSE and BER are less. And when we compare the three methods than we observed that hybrid is having good PSNR and lesser value of BER and MSE than the first two methods. As we know that for better filtering and recovering of images we need more PSNR and less BER and MSE so hybrid filtering is better than the both methods. Graphical representation of various parameters is shown in figure 10, 11 and 12. It is clear that MSE, BER are lesser for hybrid and PSNR is more for hybrid. So hybrid filtering is better than other two methods. We also concluded that different images are having different amount of MSE, BER and PSNR, according to their composition, type, size, contrast, edges and color composition etc.

\section{REFERENCES}

[1] Nikolay N. Ponomarenko, Karen O. Egiazarian, Vladimir V.Lukin, "High-Quality DCT-Based Image Compression Using Partition Schemes", IEEE Signal Processing Letters, Vol. 14, No. 2, pp.105-108, Feb. 2007.

[2] Sukhwinder Singh, Vinod Kumar, H.K. Verma, "Reduction of blocking artifacts inJPEG compressed images", Digital Signal Processing, Vol. 17, pp. 225243, Jan. 2007.

[3] Shizhong Liu, Student Member, and Alan C. Bovik, Fellow,'Efficient DCT-Domain Blind Measurement and Reduction of Blocking Artifacts", IEEE Circuits \& Systems for video Technology, Vol.12, No.12, pp.11391149, Dec. 2002

[4] F. M. Bayer and R. J. Cintra Member, "Image Compression via a Fast DCT Approximation", IEEE Latin America Transactions, Vol. 8, No. 6, pp.708-713, Dec. 2010

[5] Ee-Leng Tan1, Woon-Seng Gan1 and Meng-Tong Wong, "Fast Image Resizing in Discrete Cosine Transform Domain with Spatial Relationship between DCT Block and Its Sub-Blocks", IEEE ICALIP, Vol.1, pp. 1683-1687, July 2008.

[6] Sang-Jun Park and JechangJeong, Member, "Hybrid Image Up sampling Method in the Discrete Cosine Transform Domain", IEEE Transactions on Consumer Electronics, Vol.56, No. 4, pp.2615-2622, Nov. 2010.

[7] Wang Rui, Lin Jiangli, Li Deyu, "Edge Enhancement and Filtering of Medical Ultrasonic Images using a Hybrid Method", ICBBE Bioinformatics and Biomedical Engineering, Vol.1, pp.876 - 879, July 2007.
[8] I. O. Kirenko, R. Muijs, L. Shao, "New method for the reduction of artifacts in MPEG compressed video sequences", IEEE Multimedia \& Expo., Vol.7, No.1, pp.469-472, Dec.2006.

[9] Kawaldeep Singh,Parveen Kumar, "Algorithm for Blocking Artifact Detection \& Reduction using adaptive filtering in Compressed Images”, IJAEST, Vol. 5, No. 2, pp.156-162, 2010.

[10] Ju Jia Zou And Hong Yan, “A Deblocking Method For BDCT Compressed Images Based On Adaptive Projections", IEEE Transactions On Circuits And Systems For Video Technology, Vol. 15, No. 3, pp 430435, March 2005.

[11] M. I. Khalil, "Image Compression Using New Entropy Coder", International Journal of Computer Theory and Engineering, (1793-8201), Vol. 2, No. 1, pp39-41 February 2010

[12] M. Mitreaa, F. Preteuxb, Vlada and Fetitab, "The 2d-Dct Coefficient Statistical Behaviour A Comparative Analysis On Different Types Of Image Sequences", Journal Of Optoelectronics And Advanced Materials Vol. 6, No. 1, pp. 95-102, March 2004

[13] Janaki. R and Dr. Tamilarasi, "Enhanced ROI (Region of Interest Algorithms) for Medical Image Compression", International Journal of Computer Applications (0975 8887) Volume 38- No.2, pp 38-43, January 2012.

[14] Fang Zhu , "Blocking Artifacts Reduction in Compressed data", International Conference on Computer Engineering and Applications, IPCSIT, vol.2, pp.1-5, 2011.

[15] Stefan Winkler And Praveen Mohandas, "The Evolution Of Video Quality Measurement From PSNR To Hybrid Metrics", IEEE Trans. Broadcasting Vol. 54, No. 3, pp.19, September 2008.

[16] Dr. Anil Kumar Sharma And Yunus Mohammed Pervej, "Simulation And Analysis Of Digital Video Watermarking Using MPEG-2", International Journal On Computer Science And Engineering (IJCSE), ISSN : 0975-3397, Vol. 3, No. 7, Pp. 2700-2706 , July 2011.

[17] Dr.H.B.Kekre, "Iris recognition using Partial Coefficients by applying Discrete Cosine Transform, Haar Wavelet and DCT Wavelet Transform", International Journal of Computer Applications (0975 8887) Volume 32- No.6, pp 39-43, October 2011.

[18] V.Santhi and Dr. Arunkumar Thangavelu, "DWT-SVD Combined Full Band Robust Watermarking Technique for Color Images in YUV Color Space", International Journal of Computer Theory and Engineering, (17938201), Vol. 1, No. 4, pp.424-429, October 2009.

[19] Zhi-Heng Zhou and Sheng-Li Xie, "Sigmoid Function Activated Blocking Artifacts Reduction Algorithm", Springer-Verlag Berlin Heidelberg, K. Li, and G.W Irwin (Eds.), ICIC, LNCIS. 345, pp. 507-516, 2006. 